\title{
A novel variant in the $C L C N 1$ gene associated with dominant myotonia congenita reduces the macroscopic chloride conductance and strongly dampens its voltage dependence
}

\author{
Kevin Jehasse $^{1}$, Kathleen Jacquerie ${ }^{2}$, Alice de Froidmont ${ }^{1}$, Camille Lemoine ${ }^{1}$, Thierry \\ Grisar $^{1}$, Katrien Stouffs ${ }^{3}$, Bernard Lakaye ${ }^{1}$, and Vincent Seutin ${ }^{1}$ \\ ${ }^{1}$ Université de Liège Faculté de Médecine \\ ${ }^{2}$ Université de Liège Faculté des Sciences Appliquées \\ ${ }^{3}$ Universitair Ziekenhuis Brussel
}

April 28, 2020

\begin{abstract}
We describe a patient presenting the symptoms of myotonia congenita with a new heterozygous missense variant in exon 9 of the CLCN1 gene (c.1010T $>$ G, p.(Phe337Cys)). The mutation is located in the large extracellular loop between the I and $\mathrm{J}$ transmembrane segments of CLCN1 and we functionally analyzed its consequences on channel properties. Confocal imaging showed that the F337C mutant incorporated as well as the WT channel into the plasma membrane. Using patch clamp recordings of $\mathrm{WT}$ and $\mathrm{F} 337 \mathrm{C}$ hClC-1 channels expressed in HEK293 cells, we observed a smaller conductance for the latter at $-80 \mathrm{mV}$. Using classical voltage protocols and curve fitting procedures, we also found a marked reduction of the fast gating component in the mutant channels, as well as an overall reduced voltage-dependence. The mutation did not alter the pharmacology of the channels. Thus the loss of function is due to a reduction of the opening at resting potential and an inability to quickly activate during the action potential and protect the myocytes against repetitive discharges. To our knowledge, this is the first report of a mixed alteration in the biophysical properties of $\mathrm{hClC}-1$ consisting of a reduced conductance at resting potential and an almost abolished voltage dependence.
\end{abstract}

\section{A novel variant in the $C L C N 1$ gene associated with dominant myotonia congenita reduces the macroscopic chloride conductance and strongly dampens its voltage dependence}

Kevin Jehasse ${ }^{1}$, Kathleen Jacquerie ${ }^{3}$, Alice de Froidmont ${ }^{1}$, Camille Lemoine ${ }^{1}$, Thierry Grisar $^{2}$, Katrien Stouffs ${ }^{4}$, Bernard Lakaye ${ }^{2, *}$ and Vincent Seutin ${ }^{1, *}$

Laboratories of ${ }^{1}$ Neurophysiology and ${ }^{2}$ Molecular regulation of neurogenesis, GIGA Institute,${ }^{3}$ Department of Electrical Engineering and Computer Science, all at Liège University, 4000 Liège, Belgium, ${ }^{4}$ Vrije Universiteit Brussel (VUB), Universitair Ziekenhuis Brussel (UZ Brussel), reproduction and genetics, Neurogenetics Research Group, 1090 Brussels, Belgium.

*Both last authors contributed equally to this work

Correspondence: Vincent Seutin (V.Seutin@uliege.be). Orcid : 0000-0002-8046-8142

GRANT INFORMATION

This research was funded by grant R.FNRS.4129-9-F (KJ and VS) from the Fonds National de la Recherche Scientifique (FNRS, Belgium) and grant R.CFRA.2958 (BL) from the University of Liège (Belgium).

CONFLICT OF INTERESTS 
The authors declare no conflicts of interest with respect to the research, authorship, or publication of this article.

\section{ABSTRACT}

We describe a patient presenting the symptoms of myotonia congenita with a new heterozygous missense variant in exon 9 of the $C L C N 1$ gene $(\mathrm{c} .1010 \mathrm{~T}>\mathrm{G}$, p.(Phe337Cys)). The mutation is located in the large extracellular loop between the I and $\mathrm{J}$ transmembrane segments of $C L C N 1$ and we functionally analyzed its consequences on channel properties. Confocal imaging showed that the F337C mutant incorporated as well as the WT channel into the plasma membrane. Using patch clamp recordings of WT and $\mathrm{F} 337 \mathrm{C} \mathrm{hClC}-1$ channels expressed in HEK293 cells, we observed a smaller conductance for the latter at $-80 \mathrm{mV}$. Using classical voltage protocols and curve fitting procedures, we also found a marked reduction of the fast gating component in the mutant channels, as well as an overall reduced voltage-dependence. The mutation did not alter the pharmacology of the channels. Thus the loss of function is due to a reduction of the opening at resting potential and an inability to quickly activate during the action potential and protect the myocytes against repetitive discharges. To our knowledge, this is the first report of a mixed alteration in the biophysical properties of hClC-1 consisting of a reduced conductance at resting potential and an almost abolished voltage dependence.

\section{KEYWORDS}

CLCN1 , patch-clamp, myotonia, channel gating, microscopy

\section{INTRODUCTION}

Myotonia congenita (MC) is the most common genetic channelopathy affecting the skeletal muscle (Horga et al., 2013). Clinically, it is characterized by muscle stiffness following a voluntary movement, sometimes associated with a «warm up » phenomenon, which reflects the fact that this symptom improves with repetitive activity. By far the most frequent cause of $\mathrm{MC}$ is a pathogenic variant within the CLCN1 gene, which encodes the CLC-1 channel protein (Jentsch and Pusch, 2018). This channel is the main contributor $(\sim 80 \%)$ of the resting conductance of skeletal muscle. It normally acts to dampen myocyte excitability after an action potential. It is probably more important in this respect than $\mathrm{K}^{+}$channels that also operate at rest because, in restricted spaces such as T-tubules, $\mathrm{K}^{+}$channel openings will lead to increases in the external concentration of $\mathrm{K}^{+}$, which in turn will depolarize the muscle fiber. Because of the large volume of the myocyte, an increase in internal $\mathrm{Cl}^{-}$, which would also depolarize the myocyte, is less likely (Jentsch and Pusch, 2018).

MC can be inherited in a dominant (Thomsen's disease) or recessive form (Becker's disease). Symptomatology is usually more severe in the latter because no rescue or attenuation is possible by normal channel proteins. Indeed, it is usually considered that severe myotonia is associated with a large reduction in the ClC-1 conductance (by at least 80\%), whereas smaller reductions are supposed to yield a mild phenotype (ColdingJørgensen, 2005).

ClC-1 channels are homodimeric channels that present a double-barreled structure (Ludewig et al., 1996; Middleton et al., 1996; Mindell et al., 2001; Park and Mackinnon, 2018). Extensive biophysical analyses have demonstrated the voltage dependence of these channels, which have two protopore gates (involving the individual subunits) and one common gate. This common gate affects both pores simultaneously across an intersubunit interface and is relatively slow whereas the so-called protopore gates are faster (Accardi and Pusch, 2000). Both gates need to be open to conduct $\mathrm{Cl}^{-}$ions. The opening of both gates is voltagedependent, with an increase in open probability at potentials more depolarized than the muscle resting potential $(-80 \mathrm{mV})$. However, the open probability is not zero at $-80 \mathrm{mV}$, but about 0.6 and 0.2 , for the slow and fast gate, respectively (Accardi and Pusch, 2000). This explains why the channel both contributes to the resting conductance and is able to increase its opening probability during any depolarizing event (the time constant of the fast gate is fast enough for the ClC-1 channel to contribute to the repolarization of the action potential). 
More than 130 pathogenic variants in $C L C N 1$ have been identified, including small deletions, insertions, frame-shifts, stop codons, missense and splice-site mutations (Imbrici et al., 2015). Generally, those variants in $\mathrm{MC}$ are responsible for a defect in the channel trafficking to the membrane (Vindas-Smith et al., 2016) or a change in the biophysical properties by shifting the activation curve, reducing the unitary conductance or changing the ion selectivity (Fahlke et al., 1997; Tsujino et al., 2011; Ulzi et al., 2012; Weinberger et al., 2012; Desaphy et al., 2013). It seems that, in dominant MC, a « hot spot » of mutations is observed in or close to exon 8, encompassing the regions between loops $\mathrm{H}$ and $\mathrm{J}$ of the channel (Fialho et al., 2007). In several cases, a major depolarizing shift of the curve describing the opening of the gate versus the voltage has been described, e.g. I290M (Pusch et al., 1995) or F306L (Fialho et al., 2007). Such a shift will strongly reduce the ability of the channel to help repolarize the myocyte membrane at the end of the action potential. In other cases (e.g. A313V), an almost complete abolition of the ClC-1 current was found (Fialho et al., 2007).

Here we report on a Belgian patient suffering from dominant $\mathrm{MC}$ associated with a novel heterozygous variant $(\mathrm{F} 337 \mathrm{C})$ in the same region. Using functional analysis, we found a functional phenotype that is intermediate between those described above. We observed a reduction of the whole-cell conductance and a strong reduction in the voltage-dependence of the current.

\section{METHODS}

Informed consent of the patient

The Ethics Committee of the local institution deemed that this research did not have to be submitted to it, provided that a written agreement was obtained from the patient. This agreement was obtained by TG.

Gene sequencing

Sanger sequencing was performed for all coding exons and part of the flanking introns using standard procedures. This new variant has been submitted to ClinVar (SUB7196737).

Mutagenesis

Plasmid pRcCMV-YFP-hClC-1 was a generous gift of Dr. C. Fahlke and had been described previously (Ronstedt et al., 2015). Mutations were introduced into the plasmid by replacing a $1.1 \mathrm{~kb} \mathrm{SpeI} / \mathrm{AfeI}$ fragment by a mutated (either F337C or Q412P) double stranded gBlock DNA Fragment (IDT-DNA, Belgium). The sequences were verified by sequencing (GIGA-Genomics platform, Liège University).

Electrophysiology

Experiments were performed on transiently transfected HEK-293 cells. The cells were transfected with $1 \mu \mathrm{g}$ plasmid pRcCMV/YFP/hClC-1, containing the full-length WT hClC-1 cDNA or the F337C hClC-1, using TransITß-LT1 (Mirus Bio, Madison, WI). Cells were used $24 \mathrm{~h}$ after transfection. Whole-cell patch-clamp experiments were performed on an inverted microscope (Zeiss Axio Observer Z1) and transfected cells were visualized with fluorescent light (Zeiss Hbo 50/ac Fluorescence Mercury Light Source). Data was acquired at room temperature $\left(21-24^{\circ} \mathrm{C}\right)$ using an EPC-10 amplifier (Heka, Lambrecht, Germany) and Patchmaster software (Heka, Lambrecht, Germany). Low-resistance pipettes ([?] $3 \mathrm{M} \Omega$ ) were pulled from filamented borosilicate glass tubing (2.0 mm outer diameter, $0.42 \mathrm{~mm}$ wall thickness; Hilgenberg) with a P87 puller (Sutter Instruments, Novato, CA, USA). Recordings in which the series resistance was [?] $10 \mathrm{M} \Omega$ were discarded. The composition of the extracellular solution which superfused (@2 ml/min) the cells was (in $\mathrm{mM}$ ): $140 \mathrm{NaCl}, 4 \mathrm{KCl}, 2 \mathrm{CaCl}_{2}, 1 \mathrm{MgCl}_{2}$ and 5 HEPES, with the $\mathrm{pH}$ adjusted to 7.4 using $\mathrm{NaOH}$. The composition of the internal solution, was (in $\mathrm{mM}$ ): $140 \mathrm{NaCl}, 2 \mathrm{MgCl}_{2}, 5$ EGTA, 10 HEPES, pH adjusted to 7.4 with $\mathrm{NaOH}$. In this condition, the reversal potential of $\mathrm{Cl}^{-}$was $-1 \mathrm{mV}$. Cells were therefore held at 0 $\mathrm{mV}$.

Currents were low-pass filtered at $10 \mathrm{kHz}$. To quantify the currents flowing through CLC-1 channels, a 10 $\mathrm{mV}$ step protocol was applied from the holding potential of $0 \mathrm{mV}$ from $-200 \mathrm{mV}$ to $90 \mathrm{mV}$ for $350 \mathrm{~ms}$, followed by a step at $-100 \mathrm{mV}$ for $350 \mathrm{~ms}$ before returning to the holding potential. $1 \mathrm{mM} \mathrm{CdCl}_{2}, 1 \mathrm{mM}$ $\mathrm{ZnSO}_{4}$ and $100 \mu \mathrm{M}$ niflumic acid (NA, Tocris) were used to characterize the pharmacological properties of 
the channels. An 8-channel-perfusion system (VM8, Ala science, Westbury, NY, USA) was used to apply individual drugs locally onto the cells.

Data analysis

$\mathrm{MatLab}^{\circ}$ was used to analyze the time course of current deactivation. An algorithm was developed to fit the decay of currents elicited between -200 and $-60 \mathrm{mV}$ with a sum of two exponentials and a time-independent component (Desaphy et al., 2013). The following equation was used :

Eq.1 I (t) $=$ Afast $\cdot \exp (-\mathrm{t} / \tau$ fast $)+$ Aslow $\cdot \exp (-\mathrm{t} /$ tslow $)+\mathrm{C}$

where $\tau$ fast and $\tau$ slow are the time constants of the fast and slow components of current relaxation/deactivation, and Afast, Aslow, are the weights of the fast and slow components, and $\mathrm{C}$ is the steady-state component of the current. Relative Afast, Aslow, and $\mathrm{C}$ were defined as the ratio of the parameter on the sum of Afast, Aslow, and C.

The voltage dependence of channel activation was examined by plotting the apparent open probability $\left(\mathrm{P}_{\mathrm{o}}\right)$ as a function of the pre-pulse voltage ranging from $-200 \mathrm{mV}$ to $90 \mathrm{mV}$. The $\mathrm{P}_{\mathrm{o}}$ was calculated from the current amplitude measured at the peak of the tail current, ie. right after the cell capacitive response (Warnstedt et al., 2002) and then normalized by its maximal amplitude, ie. the current amplitude associated to the prepulse to $90 \mathrm{mV}$. From these normalized peaks of the tail current, we fitted a Boltzmann function according to the following formula,

Eq.2 $\mathrm{P}_{\mathrm{o}}(\mathrm{V})=\mathrm{Min}+(1-\mathrm{Min}) /\left[1+\exp \left(\left(\mathrm{V}_{1 / 2}-\mathrm{V}\right) / \mathrm{S}\right]\right.$

where Min is the minimal value of $\mathrm{P}_{\mathrm{o}}, \mathrm{V} 1 / 2$ is the half-maximal activation potential, and $\mathrm{S}$ is the slope factor. Both types of analysis were done on a subset of the recordings with the lowest noise in order to enhance their reliability.

Confocal imaging

HEK293 cells were plated in 24-well plates at a density of $1.810^{5}$ cells/well in DMEM (Lonza). They were transfected with either an empty plasmid or a plasmid containing cDNA of WT, F337C or Q412P channels. The latter were chosen as a control because they had previously been demonstrated to have a very small surface expression (Vindas-Smith et al., 2016). The concentration of all plasmids was $0.1 \mu \mathrm{g} / \mu \mathrm{l}$. Cells were transfected using Xtreme Gene (Sigma Aldrich), diluted at 1/33 in Mix OptiMEM and applied at room temperature for 15 minutes. On the next day, « Cellview $\gg$ dishes (Greiner Bio One) were coated with $100 \mu \mathrm{g} / \mathrm{ml}$ Poly-D-Lysine (Lonza) and left for $1 \mathrm{~h}$ at $37^{\circ} \mathrm{C}$. After rincing and trypsin (Gibco - ThermoFisher Scientific) digestion, the cells were plated $(2 \mathrm{ml} /$ dish $)$. On the next day, cells were observed using an inverted Zeiss confocal microscope (LSM 880 Elyra S1). For quantification of the fluorescence, the Fiji program was used. We first substracted the background fluorescence and measured the fluorescence of membrane portions. These experiments were done in triplicate ( 3 separate transfections). In each of the 3 experiments, $\sim 10$ cells were analyzed per genotype. In each cell, the fluorescence was quantified as one n.

Statistical analysis

Results are expressed as mean \pm SEM. Statistical analysis was performed using Prism version 8 (Graphpad Software), using the adequate statistical test. $\mathrm{P}<0.05$ was considered as significant.

\section{RESULTS}

\section{Case presentation}

Since her infancy, a 23-year old woman complained of transient muscle stiffness. She experienced difficulties mainly when initiating movements (such as when starting a walk, moving eyes, talking, or masticating). Her symptoms improved after repeated contractions, which was suggestive of a « warm up $\gg$ phenomenon. They also tended to worsen with cold, e.g. when washing her face with cold water. There was no complaint of muscle weakness after a rest. 
The patient had non-consanguineous parents. Her mother, as well as one grand uncle on the maternal side exhibited a similar semiology, suggesting a Thomsen type form of the disease. Her growth had been normal and no other disease was reported.

Medical examination showed general muscle hypertrophy, normal muscle strength and attenuated myotatic reflexes. An electroneuromyogram clearly confirmed the presence of myotonic burst discharges. Typical myotonic contractions could be clearly elicited by percussion of muscles of either the tongue or the thenar eminence. Serum creatine kinase was at 220 IU/1 (normal range: 15-130 IU/1). DNA isolated from blood lymphocytes was amplified by PCR and the 23 exons of $C L C N 1$, as well as part of the flanking introns, were sequenced. The analysis showed that the patient is a heterozygous carrier of a c.1010T $>$ G, p.(Phe337Cys) substitution in exon 9. This alteration has never been described before and was initially considered as a variant of unknown significance (VUS). In silico analysis programs (SIFT ${ }^{\circ}$, PolyPhen2 ${ }^{\circ}$, Mutation Taster ${ }^{\circ}$ and Align GVGD ${ }^{\circ}$ ) indicated that the substitution of this highly conserved amino acid (Fig. 1) is probably pathogenic, especially in view of a change from a hydrophobic amino acid to a cysteine. We therefore decided to investigate the trafficking and functionality of this mutant channel.

Subcellular localization of the F337C variant

We first investigated whether the F337C mutation affects the expression of the channel at the cell membrane. Since the channels were tagged with YFP (see construct in Methods), we evaluated their surface membrane expression by quantifying the fluorescence at the membrane. To appreciate the membrane density of F337C, we compared it with the one of WT and Q412P hClC-1 channels (Fig. 2). Indeed, the latter mutation is known to impair channel translocation to the membrane (Vindas-Smith et al., 2016). There was a significant difference between the membrane fluorescence exhibited by the three channel species (one-way ANOVA test, $\mathrm{F}=14.37,40 \mathrm{df}, \mathrm{p}<0.0001)$. Post hoc tests demonstrated that the membrane fluorescence of the F337C hClC-1 ( $\mathrm{n}=18)$ and WT hClC-1 $(\mathrm{n}=13)$ was similar (Tukey's multiple comparisons test, $\mathrm{p}=0.75)$ and much higher than the one of Q412P $(\mathrm{n}=10)$, as expected (Tukey's multiple comparisons test, $\mathrm{p}=0.0004$ for WT vs Q412P, $\mathrm{p}<0.001$ for F337C vs Q412P). Thus, the F337C mutation does not decrease the density of the channels at the membrane. We therefore turned to patch clamp recordings to determine the biophysical properties of the $\mathrm{F} 337 \mathrm{C} \mathrm{hClC}-1$.

Electrophysiological and pharmacological phenotype of the F337C mutant

We used a voltage clamp protocol to evaluate the functional properties of the $\mathrm{WT}$ and $\mathrm{F} 337 \mathrm{C}$ hClC-1 channels. In this protocol, the cells were held at $0 \mathrm{mV}$ to strongly activate the fast and slow gates. Deactivation of the channels at negative potentials could be visualized as a biexponential decrease of the current. The instantaneous and the steady state currents were measured respectively at the beginning ( $\sim \mathrm{ms})$ and the end $(\sim 350 \mathrm{~ms})$ of each pulse of voltage to obtain the IV curves shown in Fig. 3A-C. In the WT, we observed a clear deactivation of the channel in the range of $-200 \mathrm{mV}$ to $-100 \mathrm{mV}$. In F337C, the instantaneous current was much smaller in the same voltage range, reflecting the fact that the gates had not been activated at 0 $\mathrm{mV}$. When plotting the IV curves of the instantaneous currents from the WT and F337C, we observed in the mutant a loss of the typical inward rectification (Fig.3 B) of these channels. As far as the steady-state currents were concerned, F337C generated a linear current, smaller in amplitude that the one generated by the WT. In addition, the typical notch seen around $-120 \mathrm{mV}$, that was seen in the WT, was lost in the mutant, making its current extremely linear, resembling a leak channel. In order to verify that this « leaktype $\gg$ current was indeed generated by the mutant, we performed the same recordings on non-transfected cells (data not shown). No detectable current was measured. The leak conductance of the mutant around -80 $\mathrm{mV}$ (as assessed by measuring the slope of the IV curve around that voltage in the steady state condition) was significantly smaller than the one of the WT channel, $11.88 \pm 0.4 \mathrm{nS}(\mathrm{n}=17)$ vs $21.99 \pm 0.4(\mathrm{n}=26)$ (Student's t-test, $\mathrm{t}=3.779,41 \mathrm{df}, \mathrm{p}<0.001$ ), but only by about $50 \%$. The voltage dependence of the channel activation was obtained from the apparent $\mathrm{P}_{\mathrm{o}}$ as a function of the membrane voltage (Fig. 3D), which was fitted with a Boltzmann equation (Eq.2). The parameters of the fit are reported in Table 1. F337C decreased the voltage dependence of the apparent $P_{o}$ without affecting its $V_{1 / 2}$. 
To quantify the loss of current deactivation in F337C, we fitted the curves using a double exponential function as described in the methods, including the residual current, between $-200 \mathrm{mV}$ and $-60 \mathrm{mV}$. There was a significant difference between WT and F337C in the fast and slow amplitude components, as well as in the residual component (Fig. 4). Thus the progressive increase in the weight of the fast deactivation (A fast) at more negative potentials was lost in the mutant, as was the weight of the slow deactivation in the -100 to $-150 \mathrm{mV}$ range. In addition, the relative weight of the constant current was high at all voltages in the mutant, whereas it decreased towards the more negative potentials in WT channels, because of the preponderance of current deactivation.

Since the F337C mutation causes a major change in the biophysical properties of hClC-1 channels, we tested whether its pharmacological properties were altered as well. In order to do so, we tested three blockers of ClC-1 currents: niflumic acid (NA), $\mathrm{Cd}^{2+}$ and $\mathrm{Zn}^{2+}$ (Duffield et al., 2005; Liantonio et al., 2007; Yu et al., 2015). We performed the same voltage-clamp protocol. To facilitate comparison of the inhibitory effects of the drugs, currents were normalized to the instantaneous current at $-200 \mathrm{mV}$ and the degree of inhibition was obtained by the ratio of normalized current at $-200 \mathrm{mV}$ during drug application on the normalized current at $-200 \mathrm{mV}$ before drug application, subtracted from $100 \%$. The degree of inhibition by NA (100 $\mu \mathrm{M}), \mathrm{Cd}^{2+}(1 \mathrm{mM})$ and $\mathrm{Zn}^{2+}(1 \mathrm{mM})$ was not significantly different between WT and F337C for either the instantaneous current or the steady current (Mann-Whitney U test, $\mathrm{p}>0.05$ for each condition, data not shown). These results show that, even though the biophysics of the channel was markedly altered, F337C had pharmacological properties which are typical of WT hClC-1 channels.

\section{DISCUSSION}

We have described the features of a new amino acid substitution (F337C) found in a patient affected by dominant MC. This alteration significantly decreased the macroscopic conductance at the resting membrane potential (about $50 \%$ of the conductance of $\mathrm{WT} \mathrm{hClC}-1$ channels), and strongly reduced its voltage dependence. Functionally, this means that the channel is able to sustain the resting membrane potential of this patient's myocytes. However, it will not be able to massively activate during action potentials and to powerfully bring the membrane potential back to its resting value. Therefore, the likelihood of repetitive action potentials underlying myotonic burst discharges will be much increased. The clear myotonic phenotype of the patient bearing this mutation suggests that the repolarizing effect of hClC- 1 during an action potential may be more important than its resting activity to protect normal fibers from abnormal repetitive discharges, a question that is somewhat controversial (Pedersen et al., 2016). As these authors point out, however, the physiological regulation of the channel under exercise conditions by PKC, intracellular acidosis and lactate ions may modulate these effects in vivo .

To our knowledge, the phenotype of the F337C mutant is rather original in the sense that most pathogenic variants within or close to the the exon 8 hotspot (our variant is within exon 9) either almost completely abolish channel conductance (which was not the case here) or shift voltage-dependence to more depolarized potentials (whereas $\mathrm{V}_{1 / 2}$ was not changed, but voltage-dependence was reduced in our case) (Imbrici et al., 2015). Interestingly the F337C mutation is located within an extracellular loop and the phenylalanine at this position is a strictly conserved amino-acid (Fig. 1B). Three large extracellular loops link helices I-J (amino acids 322-347 based on Uniprot), helices L-M (amino acids 427-457) and helices N-O (amino acids 499-521). Among these three loops, the first one is the most conserved during evolution, with $90 \%$ identity between human and Xenopus orDanio Rerio sequences, the conservation of the two others being lower (around 50\% for the second one and $75 \%$ for the third one). As expected from their location, mutations on extracellular loops are, most of the time, less drastic than mutations within helices. One recessive variant (R453W) has been described in the second loop (Brugnoni et al., 2013) but it does not affect a conserved residue and had no effect on channel properties (Portaro et al., 2015). In the third loop, the L520P mutation was reported in one patient but its consequences on channel properties are unknown (this residue is highly conserved) (Brugnoni et al., 2013). In the first loop, 4 other residues were reported as mutated in some MC patients. Mutation V327I is recessive and its consequences on channel properties have not been studied but it may impair splicing, and by consequence channel production, as the nucleotide substitution is within the consensus 
splice site (Horga et al., 2013). The I329T mutation is recessive and it shifts the voltage dependence of open probability to the right but also affects channel deactivation (Zhang et al., 2000). The mode of inheritance of A331T is unknown and this mutation produces complex effects on the gating (Warnstedt et al., 2002). Finally two mutations have been reported for residue R338, one nonsense (Brugnoni et al., 2013) and one missense (R338Q). The R338Q mutation can be dominant or recessive and shifts the voltage dependence of the open probability by $38 \mathrm{mV}$ to the right (Zhang et al., 2000). The phenotype of the F337C mutant is therefore rather original in the sense that its voltage dependence is strongly reduced. The question arises whether this phenotype may be due to the cysteine residue of the mutant channel. It is possible that this replacement will yield an abnormal intramonomer or intermonomer disulfide bridge which would be responsible for this specific channel phenotype. The smaller voltage-dependence of the $\mathrm{P}_{\mathrm{o}}$ could be due to an alteration of one of the two gates, as is the case for G233S (Richman et al., 2012) or both. Further experiments using reducing conditions, alternative substitutions of the phenylalanine and measuring the $\mathrm{P}_{\mathrm{o}}$ of the fast and slow gating separately will help answer this question.

On the other hand, the pharmacology of the mutant channel was not affected. This is not surprising. For example, niflumic acid is supposed to block hClC-1 at least in part by binding to the internal mouth of the channel (Liantonio et al., 2007), which is unlikely to be affected in this mutant.

In summary, we have found a novel mutant of the hClC-1 channel protein, F337C, that is most likely responsible for the myotonic symptomatology of this patient. When expressed in HEK293 cells, this mutant inserts normally in the membrane, but has major functional alterations consisting in a reduced steady-state conductance and a loss of voltage dependence.

\section{ACKNOWLEDGMENTS}

This research was funded by grant R.FNRS.4129-9-F (KJ and VS) from the Fonds National de la Recherche Scientifique (FNRS, Belgium) and grant R.CFRA.2958 (BL) from the University of Liège (Belgium).We thank C.Gillissen, P. Shah and B. Coumans for their technical help and the patient for her willingness to contribute to the knowledge of

\section{CLCN1.}

\section{REFERENCES}

Accardi A, Pusch M. 2000. Fast and slow gating relaxations in the muscle chloride channel CLC-1. J Gen Physiol 116:433-444.

Brugnoni R, Kapetis D, Imbrici P, Pessia M, Canioni E, Colleoni L, Rosbo NK De, Morandi L, Cudia P, Gashemi N, Bernasconi P, Desaphy JF, et al. 2013. A large cohort of myotonia congenita probands: Novel mutations and a high-frequency mutation region in exons 4 and 5 of the CLCN1 gene. J Hum Genet $58: 581-587$.

Colding-Jørgensen E. 2005. Phenotypic variability in myotonia congenita. Muscle Nerve 32:19-34.

Desaphy J-F, Gramegna G, Altamura C, Dinardo MM, Imbrici P, George AL, Modoni A, Lomonaco M, Conte Camerino D, Camerino DC. 2013. Functional characterization of ClC-1 mutations from patients affected by recessive myotonia congenita presenting with different clinical phenotypes. Exp Neurol 248:530-40.

Duffield MD, Rychkov GY, Bretag AH, Roberts ML. 2005. Zinc inhibits human ClC-1 muscle chloride channel by interacting with its common gating mechanism. J Physiol 568:5-12.

Fahlke C, Beck CL, George AL. 1997. A mutation in autosomal dominant myotonia congenita affects pore properties of the muscle chloride channel. Proc Natl Acad Sci U S A 94:2729-2734.

Fialho D, Schorge S, Pucovska U, Davies NP, Labrum R, Haworth A, Stanley E, Sud R, Wakeling W, Davis MB, Kullmann DM, Hanna MG. 2007. Chloride channel myotonia: exon 8 hot-spot for dominant-negative interactions. Brain 130:3265-74. 
Horga A, Rayan DLR, Matthews E, Sud R, Fialho D, Durran SCM, Burge JA, Portaro S, Davis MB, Haworth A, Hanna MG. 2013. Prevalence study of genetically defined skeletal muscle channelopathies in England. Neurology 80:1472-1475.

Imbrici P, Altamura C, Pessia M, Mantegazza R, Desaphy JF, Camerino DC. 2015. CIC-1 chloride channels: State-of-the-art research and future challenges. Front Cell Neurosci 9:.

Jentsch TJ, Pusch M. 2018. CLC chloride channels and transporters: Structure, function, physiology, and disease. Physiol Rev 98:1493-1590.

Liantonio A, Giannuzzi V, Picollo A, Babini E, Pusch M, Conte Camerino D. 2007. Niflumic acid inhibits chloride conductance of rat skeletal muscle by directly inhibiting the CLC-1 channel and by increasing intracellular calcium. Br J Pharmacol 150:235-247.

Ludewig U, Pusch M, Jentsch TJ. 1996. Two physically distinct pores in the dimeric CIC-0 chloride channel. Nature 383:340-343.

Middleton RE, Pheasant DJ, Miller C. 1996. Homodimeric architecture of a CIC-type chloride ion channel. Nature 383:337-340.

Mindell JA, Maduke M, Miller C, Grigorieff N. 2001. Projection structure of a CIC-type chloride channel at $6.5 \AA$ A resolution. Nature 409:219-223.

Park E, Mackinnon R. 2018. Structure of the CLC-1 chloride channel from homo sapiens. Elife 7:e36629.

Pedersen TH, Riisager A, Paoli FV de, Chen T-Y, Nielsen OB. 2016. Role of physiological ClC-1 Cl- ion channel regulation for the excitability and function of working skeletal muscle. J Gen Physiol 147:291-308.

Portaro S, Altamura * Concetta, Licata N, Camerino GM, Imbrici P, Musumeci O, Rodolico C, Conte Camerino D, Toscano A, Desaphy J-F. 2015. Clinical, Molecular, and Functional Characterization of CLCN1 Mutations in Three Families with Recessive Myotonia Congenita. NeuroMolecular Med 17:285-296.

Pusch M, Steinmeyer K, Koch MC, Jentsch TJ. 1995. Mutations in dominant human myotonia congenita drastically alter the voltage dependence of the CIC-1 chloride channel. Neuron 15:1455-1463.

Richman DP, Yu Y, Lee TT, Tseng PY, Yu WP, Maselli RA, Tang CY, Chen TY. 2012. Dominantly inherited myotonia congenita resulting from a mutation that increases open probability of the muscle chloride channel CLC-1. NeuroMolecular Med 14:328-337.

Ronstedt K, Sternberg D, Detro-Dassen S, Gramkow T, Begemann B, Becher T, Kilian P, Grieschat M, Machtens JP, Schmalzing G, Fischer M, Fahlke C. 2015. Impaired surface membrane insertion of homo- and heterodimeric human muscle chloride channels carrying amino-terminal myotonia-causing mutations. Sci Rep 5:15382.

Tsujino A, Kaibara M, Hayashi H, Eguchi H, Nakayama S, Sato K, Fukuda T, Tateishi Y, Shirabe S, Taniyama K, Kawakami A. 2011. A CLCN1 mutation in dominant myotonia congenita impairs the increment of chloride conductance during repetitive depolarization. Neurosci Lett 494:155-160.

Ulzi G, Lecchi M, Sansone V, Redaelli E, Corti E, Saccomanno D, Pagliarani S, Corti S, Magri F, Raimondi M, D'Angelo G, Modoni A, et al. 2012. Myotonia congenita: Novel mutations in CLCN1 gene and functional characterizations in Italian patients. J Neurol Sci 318:65-71.

Vindas-Smith R, Fiore M, Vasquez M, Cuenca P, Valle G del, Lagostena L, Gaitan-Penas H, Estevez R, Pusch M, Morales F. 2016. Identification and Functional Characterization of CLCN1 Mutations Found in Nondystrophic Myotonia Patients. Hum Mutat 37:74-83.

Warnstedt M, Sun C, Poser B, Escriva MJ, Tranebjaerg L, Torbergsen T, Ghelue M Van, Fahlke C. 2002. The Myotonia Congenita Mutation A331T Confers a Novel Hyperpolarization-Activated Gate to the Muscle Chloride Channel ClC-1. J Neurosci 22:7462-7470. 
Weinberger S, Wojciechowski D, Sternberg D, Lehmann-Horn F, Jurkat-Rott K, Becher T, Begemann B, Fahlke C, Fischer M. 2012. Disease-causing mutations C277R and C277Y modify gating of human ClC-1 chloride channels in myotonia congenita. J Physiol 590:3449-3464.

Yu Y, Tsai MF, Yu WP, Chen TY. 2015. Modulation of the slow/common gating of CLC channels by intracellular cadmium. J Gen Physiol 146:495-508.

Zhang J, Bendahhou S, Sanguinetti MC, Ptaček LJ. 2000. Functional consequences of chloride channel gene (CLCN1) mutations causing myotonia congenita. Neurology 54:937-942.

\section{LEGENDS OF THE FIGURES}

\section{Figure 1. Localization of the F337C mutation in hClC-1}

A. Schematic structure of a hClC-1 channel subunit. The F337C mutation is located in the extracellular loop between I and J transmembrane domains. CBS is the cystathionine $\beta$-synthase domain at which ATP binds ClC-1 and inhibits the common gating. B.Alignment of the amino acid sequence of the I-J extracellular loop of ClC-1 from different species shows that F337 (in bold green) is strictly conserved. Residues different from the human sequence are in bold red.

\section{Figure 2. Subcellular localization of WT, F337C and Q412P hClC-1 channels}

A. Visualization of YFP-tagged WT, F337C or Q412P hClC-1 channels in transfected HEK293 cells (scale bar: $5 \mu \mathrm{m}$ ). Note the clear membrane localization of WT and F337C channels, but not Q412P channels B.Bar charts showing the level of membrane fluorescence of WT, F337C and Q412P hClC-1. Data are shown as mean \pm SEM (arbitrary units).

\section{Figure 3. Voltage dependence of WT and F337C hClC-1 channels}

$A$. Chloride currents were recorded from HEK 293 cells transfected with either WT or F337C h-CLCN1 DNA. The protocol used is shown in the upper panel. Note the clearly reduced voltage-dependence of the F337C mutant, as shown by an almost abolished deactivation at negative potentials. $B$ and $C$. Current-voltage curves of the instantaneous and steady state hClC-1 currents, respectively, measured at the time indicated in the Methods. Instantaneous WT currents display an inward rectification whereas F337C currents are linear. Steady state F337C currents are more linear than WT currents. D. Apparent $\mathrm{P}_{\mathrm{o}}$-voltage curve. The apparent $\mathrm{P}_{\mathrm{o}}$ was obtained by measuring the tail current at $-100 \mathrm{mV}$ (post-pulse) as a function of the voltage reached during the previous step. The $\mathrm{P}_{\mathrm{o}}$ of F337C is much less voltage-dependent than that of WT. All values are represented as mean $\pm \mathrm{SEM}$

Figure 4. Kinetics of deactivating currents for WT and F337C hClC-1 channels.

The parameters of the double exponential function (see Eq.1 in the Methods) for the fit consisted of a fast and slow time constant ( $\tau$ fast and $\tau$ slow) with their respective contribution to the whole current (Afast and Aslow), in addition to the contribution of the residual current (relative C). Each point is represented as a mean \pm SEM of 9 and 12 fits for WT and F337C currents, respectively. Note the reduction of the contribution of Afast and Aslow in F337C channels.

\section{DATA AVAILABILITY STATEMENT}

The data that support the findings of this study are available from the corresponding author upon reasonable request.

\section{Hosted file}

Figures_Jehasse et al.pdf available at https://authorea.com/users/307635/articles/438635-a-novelvariant-in-the-clcn1-gene-associated-with-dominant-myotonia-congenita-reduces-the-macroscopicchloride-conductance-and-strongly-dampens-its-voltage-dependence 